\section{Livraisons}

d'Histoire

de l'Architecture

\section{Livraisons de l'histoire de l'architecture}

$25 \mid 2013$

Architectures médiévales : types, matières et formes

\title{
« Tendre » et « destendre » : l'ameublement des résidences royales à la fin du Moyen Âge
}

"Tendre" and "destendre": the furnishing of the royal residencies at the end of the $15^{\text {th }}$ century

"Tendre et destendre”, die Einrichtung der königlichen Schlösser am Ende des

Mittelalters

\section{Caroline Vrand}

\section{OpenEdition}

\section{Journals}

Édition électronique

URL : http://journals.openedition.org/lha/310

DOI : 10.4000/lha. 310

ISSN : 1960-5994

Éditeur

Association Livraisons d'histoire de l'architecture - LHA

Édition imprimée

Date de publication : 10 juin 2013

Pagination : 109-116

ISSN : 1627-4970

Référence électronique

Caroline Vrand, « «Tendre » et « destendre » : l'ameublement des résidences royales à la fin du Moyen Âge », Livraisons de l'histoire de l'architecture [En ligne], 25 | 2013, mis en ligne le 10 juin 2015, consulté le 01 mai 2019. URL : http://journals.openedition.org//ha/310; DOI : 10.4000//ha.310

Ce document a été généré automatiquement le 1 mai 2019.

Tous droits réservés à l'Association LHA 


\section{«Tendre » et « destendre »:}

\section{l'ameublement des résidences royales à la fin du Moyen Âge}

"Tendre" and "destendre": the furnishing of the royal residencies at the end of the $15^{\text {th }}$ century

"Tendre et destendre", die Einrichtung der königlichen Schlösser am Ende des

Mittelalters

Caroline Vrand

1 L'ameublement des châteaux royaux est un enjeu primordial pour la cour royale qui se met en scène à travers l'exposition de ses collections d'objets d'art, à la fin du Moyen Âge essentiellement l'orfèvrerie et les textiles. L'architecture devient alors un écrin destiné à magnifier la grandeur royale. Cet aspect est bien sûr particulièrement frappant lors des grands événements de la vie de cour, comme les naissances ou les réceptions solennelles, mais il s'agit bien d'une réalité et d'un souci quotidiens.

2 Le caractère itinérant de la cour de France, la fréquence des déplacements et la multiplicité des lieux de résidence nécessitaient toute une organisation en coulisses afin de rendre possible cette représentation quasi permanente. Si les résidences n'étaient pas complètement vidées à chaque départ de la cour, le couple royal se déplaçait néanmoins avec une partie de ses collections et s'instaurait donc un va-et-vient constant entre les différents châteaux, les logis devant être prêts et «tendus " - revêtus de tentures - à l'arrivée de la cour. Les comptes d'Anne de Bretagne, mais aussi tout particulièrement ses inventaires conservés à la Bibliothèque nationale de France ${ }^{1}$ offrent une plongée dans le quotidien des collections et leurs déplacements incessants. Il s'agit, en effet, plutôt de registres de gestion, tenus par les officiers en charge des collections, qui rendent compte au jour le jour des déplacements des effets personnels de la reine. Bien que ne soient parvenus jusqu'à nous seulement des fragments, ils permettent d'appréhender les modalités de la gestion d'une collection royale à la fin du Moyen Âge. 
3 De l'étude de la gestion des collections d'Anne de Bretagne ressort la prééminence des tapissiers de la reine. On sait que le terme de tapissiers, largement polysémique, désigne l'ensemble des artisans ou artistes qui concourrent à la réalisation d'une tapisserie, mais aussi les officiers chargés de l'entretien des collections textiles. Leur rôle auprès d'Anne de Bretagne va encore plus loin. L'officier de l'hôtel de la reine le plus souvent sollicité pour la gestion de ses collections est en effet un tapissier, Jean Lefèvre, évoqué dans pas moins de sept inventaires pour avoir eu la garde temporaire de diverses pièces textiles et, dans un huitième, celle d'orfèvrerie religieuse. Qu'il ait eu la charge de telles pièces d'orfèvrerie témoigne de son rôle prééminent au service de la reine : ses tâches, outre l'entretien des textiles, recouvrent aussi la garde de l'ensemble des collections de la reine. De plus, il est également sollicité pour assurer le transport des collections : en janvier 1498, il est chargé d'envoyer à Nantes un ensemble de tapisseries ${ }^{2}$; en janvier 1500 , plusieurs tapisseries venues de Milan et d'autres textiles et habits liturgiques ${ }^{3}$. L'importance de Jean Lefèvre est telle qu'il signe lui-même à plusieurs reprises les inventaires ${ }^{4}$, détenteur ainsi de la capacité à être caution juridique. Si Jean Lefèvre joue un rôle majeur auprès d'Anne, elle s'est aussi entourée d'autres tapissiers, dont il convient d'apprécier l'implication dans la gestion de ses collections.

Deux états des gages des officiers de l'hôtel d'Anne de Bretagne donnent les noms des tapissiers au service de la reine. Aux côtés de Jean Lefèvre, sont mentionnés pour l'année 1492-1492 Antoine Boutet, Jean de Dol et Baudichon Duhamel', et pour l'année 1498-1499 à nouveau Jean de Dol et Baudichon Duhamel ${ }^{6}$. Ces deux derniers apparaissent également dans les inventaires de la reine, cités pour être spécifiquement chargés d'assurer le transport, quand la reine "va par pays ", d'un ensemble de textiles composé de quatorze pièces de tapisseries, deux tapis, six pièces d'apparat de lit de drap d'or, deux coussins de velours et un ciel de taffetas avec ses trois rideaux assortis ${ }^{7}$. Il semble qu'il s'agit ici de tentures d'apparat de la chambre de la reine qu'elle emporte dans ses déplacements et il n'est pas anodin de constater que ces pièces de prestige sont confiées à des tapissiers. Par ailleurs, Baudichon Duhamel a également été sollicité pour porter une pièce de tapisserie de Blois à Nantes ${ }^{8}$, à une date inconnue. Selon les comptes de la Trésorerie générale de la reine, Baudichon Duhamel loue une maison dans la ville de Lyon pour y entreposer et « estuyer ${ }^{9}$ la tappicerie de ladite dame durant deux mois entiers $»^{10}$ et il est demandé à Jean de Dol de porter la chaise de la reine lors de ses dîners de mars à juin 1494 et de faire mettre à Villefranche une courroie sur «le bahut de la tapisserie de la chambre de la reine $^{11}$ " ainsi que d'en faire coudre les quatre coins ${ }^{12}$. Même si ces tapissiers ne semblent pas avoir joué un rôle aussi important et régulier que Jean Lefèvre dans la garde des collections, ils prennent pleinement part au quotidien de la vie de cour itinérante, chargés d'accompagner la reine dans ses déplacements et d'assurer le transport d'une partie de ses effets personnels. Quant à Antoine Boutet, il est intéressant de voir qu'il est désigné indifféremment comme "tapissier » et comme "coffretier-malletier » dans les comptes de la Trésorerie générale de la reine ${ }^{13}$. Aussi surprenant qu'il puisse paraitre, ce changement d'appellation est en fait révélateur des fonctions de certains tapissiers au sein de l'hôtel royal, qui supervisent, lors des déplacements de la cour, les déplacements des collections, alors entreposées dans des coffres.

5 À côté de ces tapissiers qui appartenaient à l'hôtel de la reine, il faut en mentionner d'autres qui apparaissent ponctuellement dans les inventaires mais ne font pas partie du service de la reine. On voit notamment des tapissiers du roi intervenir à deux reprises seulement dans la gestion des collections de la reine. Au mois de janvier 1502, à Blois, 
plusieurs articles sont ainsi laissés à « Robert, tapissier du roy ${ }^{14}$. Certaines tapisseries sont également confiées à un certain Gabriel, selon un inventaire du 6 septembre $1507^{15}$; il s'agit probablement de Gabriel de Herbaumez, tapissier du roi. Enfin, un autre tapissier est mentionné dans les comptes de l'année 1495-1496, mais il n'est répertorié comme tapissier de la reine dans aucun état des gages de l'hôtel conservés : il s'agit de Julien Cochet, "tappicier et garde de la tappicerie des couches et gesines de ladite dame", rémunéré pour " descharger et descendre des basteaulx la tappicerie de la dame /.../ et icelle monter et acoustrer en une haulte chambre pour mieulx la monstrer au roy et a icelle dame $»^{16}$. Il est ici clair que le tapissier participe pleinement à l'aménagement des résidences royales : c'est à lui que revient parfois la charge de «tendre » les pièces avant l'arrivée de la cour.

Ce sont donc bien les tapissiers qui sont au premier chef sollicités pour la garde, le transport et l'aménagement des collections. Parmi les nombreux tapissiers au service de la reine, il faut néanmoins souligner qu'un d'entre eux se détache tout particulièrement, par la fréquence avec laquelle il apparaît et l'importance de ses missions. Jean Lefèvre, loin d'être essentiellement employé à la manutention des textiles, apparaît bien plus comme un véritable gestionnaire de l'ensemble des collections de la reine. À cet égard, la mise en exergue du rôle de Jean Lefèvre au sein de la cour d'Anne de Bretagne remet en cause la conception selon laquelle la responsabilité des meubles royaux était l'affaire exclusive du valet de chambre ${ }^{17}$. Si Jean Lefèvre est une fois mentionné comme « varlet de chambre et premier tappicier de la reyne » dans un registre de janvier 1500, c'est là la seule occurrence de ce type et il est bien mentionné à toutes les autres comme tapissier uniquement; dans les états des gages des officiers de l'hôtel de la reine conservés, il compte bien parmi les tapissiers et non parmi les valets de chambre, qui semblent de manière générale relativement absents de la gestion des collections de la reine. Jean de Paris, peintre et valet de chambre à la fois du roi et d'Anne, plus connu sous le nom de Jean Perréal, a été une seule fois chargé de garder des biens de la reine - des pièces de vaisselle - en juin 1505. Quant aux autres valets de chambre cités dans l'état des gages de l'hôtel de la reine des années 1492-1493 - Pierre Bay, Bernard Prevost, Vincent Guito, Guillaume Rollant, Guillaume Bongar, Guillaume Porchier et Claude de la Bausnie ${ }^{18}$ - ils ne se retrouvent pas dans les inventaires de la reine et on ne peut donc rattacher aucun d'eux à une activité de gestion des collections de la reine.

7 Le cas de Jean Lefèvre permet aussi de constater que le tapissier n'est pas affecté spécifiquement à une résidence. Il est certes présenté comme "demourant a Amboise » dans les comptes de la reine et les inventaires le montrent effectivement souvent actif à Amboise. Cependant il apparaît aussi à Blois où, en en août 1500, lui sont ainsi confiés " un lict de camp et autres choses appartenant à la reyne» et, au début du mois de décembre de la même année, lui sont donnés en garde divers ornements d'église. A l'époque d'Anne de Bretagne, les tapissiers sont donc amenés à se déplacer au gré des impératifs de la reine et de la cour. Une seule mention évoque une certaine spécialisation des tapissiers. Il s'agit de Julien Cochet désigné comme «tappicier et garde de la tappicerie des couches et gesines de ladite dame ${ }^{19}$. Cependant cette mention constitue un hapax. Les tapisseries et textiles employés lors des accouchements de la reine bénéficieraient-ils d'un traitement de faveur pour avoir leur garde attitré ? Ce titre est d'autant plus problématique que c'en est la seule occurrence : les comptes des officiers de l'hôtel de la reine ne font aucunement référence ni à ce tapissier précisément, ni à un titre semblable. 
8 Enfin, les inventaires de la reine permettent de comparer l'évolution d'une carrière de tapissier à la fin du Moyen Âge et quelques décennies plus tard, sous François I ${ }^{\text {er }}$. Stéphane Castelluccio $^{20} \mathrm{a}$ en effet montré que, sous le règne de François I ${ }^{\mathrm{er}}$, l'aboutissement de la carrière d'un tapissier est de devenir concierge d'une résidence royale, la nomination au château du Louvre étant la plus prestigieuse. Or, l'étude des inventaires de la reine ne permet pas de conclure qu'une telle carrière existât déjà sous Anne de Bretagne. Bien qu'ils couvrent une période relativement longue, de 1492 à 1507, nous ne voyons aucun tapissier devenir concierge de château. Jean Lefèvre, par exemple, mentionné comme tapissier de la reine dès un inventaire du mai 1494, l'est encore en 1501 et le demeure jusqu'à sa mort, puisqu' il est ailleurs désigné comme "en son vivant tapissier de la reine $»^{21}$. Inversement, aucun concierge n'a jamais été appelé tapissier.

9 La charge de concierge de résidence royale est pourtant déjà à l'époque d'Anne de Bretagne un des éléments essentiels à la bonne gestion des collections et au suivi logisitique des incessants déplacements ${ }^{22}$. Le concierge s'occupe spécifiquement du mobilier de la résidence dont il est responsable, garde ce qui reste sur place lors des absences du couple royal et veille à ce que tout soit en place pour son retour ${ }^{23}$. Différents concierges sont cités dans nos inventaires: Louis Challon, concierge du château d'Amboise et Roccau Peignac, concierge de celui de Nantes. Un troisième est mentionné : Pierre Forget « concierge du logey neuf d'Amboise ». Plusieurs concierges peuvent donc être affectés à la même résidence : pour le château d'Amboise, il semble ainsi qu'il y ait un concierge général, Louis Challon et d'autres plus spécifiquement en charge d'une partie de la résidence, dont Pierre Forget pour le «logis neuf $»^{24}$. Un inventaire du 11 décembre 1500 répertorie les meubles et « ustencilles » conservés à Amboise et mis en garde auprès de Louis Challon, essentiellement des lits, châlits, des chaises, des draps, des ciels, des couvertures $^{25}$. C'est au même Louis Challon que revient la responsabilité de ce qui avait été laissé dans la garde-robe quand Charles VIII part pour Naples ${ }^{26}$. Quant à Pierre Forget, il reçoit, à une date non précisée, la garde de deux rideaux et de deux pièces de tapisserie à personnages restées au château d'Amboise ${ }^{27}$. Pour ce qui est du château de Nantes, il était confié à Roceau Paignac, qui a notamment dû veiller sur plusieurs pièces de textiles, linceuls de toile, couvre-chefs, toiles d'oreillers, des serges de chiffon ${ }^{28}$. Les comptes de l'année 1497-1498 indiquent aussi que la «maison d'Estampes a Paris ${ }^{29}$ dispose également d'un concierge, Gillet Rebours, qui s'est occupé de l'entretien des jardins de cette maison durant la présence de la reine. Même si cela n'est pas précisé, on peut estimer qu'il a également veillé sur les meubles et autres objets de la reine. Si les inventaires fournissent les noms de concierges de deux châteaux seulement, on peut cependant supposer que toutes les résidences royales avaient à leur tête un tel officier. Et il est fort probable que les concierges travaillaient en étroite collaboration avec les tapissiers afin de préparer les salles pour la venue de la cour. Il convient aussi de noter que la fonction de concierge ne concerne pas exclusivement les résidences royales. Le concierge de l'hôtel archiépiscopal de Lyon $^{30}$, Girard de la Roche, est également nommé dans les comptes de la reine comme «menuysier et concierge de l'ostel archiepiscopal de Lyon ». Il reçoit une rémunération pour avoir "dressé une table » dans la chambre des filles d'honneur de la reine, avoir " habillé » trois châlits - c'est à dire orner de tentures des lits - et avoir posé un marchepied le long du lit de la chambre de la reine. On sait qu'il effectue ces tâches en juillet 1494, date à laquelle la reine n'est pas encore à Lyon, où elle n'arrive qu'en août. Sa mission est donc bien de préparer les pièces de la résidence avant l'arrivée de la cour. 
10 Notons ici que parfois certaines femmes de l'entourage de la reine ont pu se substituer aux concierges. Ainsi alors qu'étonnamment aucun concierge n'est mentionné pour le château de Blois, les sources montrent que c'est souvent aux femmes qu'étaient confiés des objets laissés à Blois au départ du couple royal. En janvier 1502, Jeanne Mauray, femme de chambre de la reine, reçoit les clés de trois coffres placés dans « la chambre de la tappisserie du chateau de Blois $»^{31}$, dans lesquels avaient été rangés les linges neufs commandés pour la venue de Philippe le Beau, archiduc d'Autriche, et sa femme Jeanne de Castille en décembre 1501. En mai 1502, la reine part pour Lyon et plusieurs pièces, dont de riches objets d'orfèvreriie ${ }^{32}$, sont laissées à Blois, entreposées dans deux coffres " en la salle pres de la chambre de la reine "; leur garde est alors confiée à la nourrice d'Anne de Bretagne ${ }^{33}$. Dans un inventaire du 6 septembre 1507, on apprend que c'est encore elle qui a la clé d'un coffre contenant des "tappicerie de drap d'or ", à nouveau des pièces très luxueuses donc, et placées «en la garderobbe audit Bloys». Lorsque la cour s'absente, les tentures qui ornent les murs sont décrochées et rangées à l'abri dans des coffres. D'après ces mentions, aucune pièce n'est spécifiquement destinée à conserver ces coffres, qui sont placés tantôt dans la garde-robe, tantôt dans la salle ou d'autres pièces ${ }^{34}$.

11 Il faut enfin s'attarder sur différents lieux utilisés par Anne de Bretagne comme gardesmeubles, où elle entreposait les meubles et pièces dont elle n'avait pas une utilité immédiate. C'est seulement partir du règne de François $\mathrm{I}^{\mathrm{er}}$, de 1527 exactement, que la royauté dispose d'un bâtiment précis qui lui sert de garde-meubles des biens royaux, l'hôtel parisien du connétable de Bourbon, et ce jusqu'au milieu du XVIII ${ }^{\text {ème }}$ siècle $^{35}$. Pour Louis Douët D'Arcq, la maison de l'argentier ${ }^{36}$ était, au XIV ${ }^{\text {ème }}$ siècle du moins, «à peu près l'ancien Garde-Meuble de la couronne $»^{37}$. L'argentier d'Anne de Bretagne, Victor Gaudin $^{38}$, est pourtant assez peu présent dans les sources ${ }^{39}$ et ne semble pas de fait intervenir de manière régulière dans la gestion des collections de la reine ${ }^{40}$. Il apparaitt en réalité que la reine a eu recours à plusieurs autres lieux, qui ont fait, eux, office de gardesmeubles personnels. Le château de Nantes a ainsi visiblement été un lieu primordial à cet égard. Les inventaires montrent que la reine y entrepose à de nombreuses reprises certains de ses biens les plus luxueux et que, logiquement, c'est de Nantes qu'elle fait venir ses plus belles pièces pour des occasions particulières, comme la réception de l'archiduc d'Autriche à Blois en décembre $1501^{41}$. La maison personnelle du financier Jacques de Beaune, à Tours, a également été utilisée par Anne de Bretagne. C'est là qu'elle entrepose une grande partie de ses collections ${ }^{42}$ entre la mort du roi Charles VIII et son retour en Bretagne. La reine continue à y avoir recours sous Louis XII : un inventaire de février 1500 indique que plusieurs luxueuses pièces de vaiselle d'or y sont conservées ${ }^{43}$; un autre de juillet 1499 recense un ensemble de livres appartenant à la reine et pareillement entreposés chez lui ${ }^{44}$. Cela est d'autant plus remarquable que Jacques de Beaune, depuis 1495, ne faisait plus partie de l'hôtel de la reine. Il s'agit donc là de relations strictement personnelles et non d'une mission inhérente à une charge. Enfin, la maison de Raymond de Dezest, bailli d'Amboise, a également pu faire office de gardemeuble temporaire: plusieurs ornements d'église apportés de Naples y sont déposés, rangés dans un coffre, du 3 au 15 décembre 1500, avant de partir pour Blois. Un inventaire non daté fait à Amboise recense également des « reliquaires, lampes, custodes, chasubles, parmemens d'autel et autres choses estans demourez entre les mains de Raymond Dezest, bailly d'Amboise " sans qu'il soit précisé si ces objets sont restés au château d'Amboise sous sa garde ou s'ils ont été directement conservés chez lui. Au fil des 
inventaires, d'autres pièces sont simplement présentées comme «en la garde dudit Raymond Dezest ». Quoi qu'il en soit, Raymond de Dezest, comme Jacques de Beaune, a à plusieurs reprises été chargé de la garde des collections de la reine alors que cela ne relevait a priori pas de sa fonction : en tant que bailli d'Amboise, il était chargé de la ville et non du château, même s'il est vrai qu'il en a longtemps supervisé les travaux. Comme Jacques de Beaune, là encore, il est frappant de noter que c'est dans sa propre maison que sont parfois entreposés les objets de la reine, maison qui devient alors une sorte de gardemeubles personnel de la reine.

Pour conclure, il apparaît en réalité qu'aucune règle n'était établie a priori dans la gestion des collections à l'époque d'Anne de Bretagne. L'étude de la gestion de ces collections montre en effet surtout l'importance des relations et des choix personnels. Les attributions sont fixées davantage en fonction des officiers que des offices. Le tapissier Jean Lefèvre que nous avons vu jouer un rôle de premier plan dans la gestion de ses collections n'est autre qu'un officier breton qui était déjà au service de son père le duc François II de Bretagne. Anne semble également avoir noué des liens de confiance avec Jacques de Beaune, au point d'entreposer dans sa maison pendant plusieurs mois une grande partie de ses collections. Cela montre que la situation était assez peu codifiée à la fin du Moyen Âge et que la personne et les liens de confiance comptaient autant sinon plus que les titres officiels. Enfin, Anne semble avoir tout particulièrement utilisé de son château natal de Nantes comme garde-meuble personnel, y entreposant ses pièces les plus luxueuses et affirmant ainsi son attachement à sa Bretagne natale.

Cette gestion relativement peu institutionnalisée découle en fait directement du statut même des collections royales qui sont à cette époque elles aussi personnelles. La notion de biens de la Couronne n'existe pas encore. Preuve en est le contrat de mariage entre Charles VIII et Anne de Bretagne qui prévoit que si le roi mourait avant son épouse, celleci deviendrait propriétaire de tous ses meubles et joyaux, ce que confirment les inventaires de la reine puisqu'on retrouve en effet dans ceux d'après 1498 de nombreuses pièces directement rattachées à Charles VIII. Dans ce contexte, Anne de Bretagne a pu imprimer la marque de sa personnalité en choisissant de s'appuyer sur des hommes de confiance et des lieux de prédilection et a su affirmer par là une large autonomie.

\section{NOTES}

1. . Ms fr. 22335. En partie édités par Antoine Jean Victor Le Roux de Lincy dans Vie de la reine Anne de Bretagne: femme des rois de France Charles VIII et Louis XII, suivie de lettres inédites et de documents originaux, vol 4 : Inventaires de la reine, Paris, L. Curmer, 1860-1861.

2. . BnF ms fr. 22335, p. 129.

3. Ibid., p. 131.

4. . C'est le cas pour un inventaire du 16 août 1498 fait à Tours, un du 11 janvier 1500 et un du 29 janvier de la même année (BnF ms fr. 22335, p. 27, 51 131).

5. . Arch. nat., KK 82, fol. 55-56.

6. . Arch. dép. Loire-Atlantique, E 210 / 19.

7. . Inventaire fait à Blois le 6 septembre 1507, BnF ms fr. 22335, p. 247. 
8. . BnF ms fr. 22335, p. 250 (inventaire du 6 septembre 1507).

9. . Estuyer signifie enfermer.

10. . Arch. nat., KK 84, fol. 112.

11. Il s'agit du coffre qui renferme les tentures de la chambre de la reine.

12. . Arch. nat., KK 84, fol. $115 \mathrm{v}^{\circ}$.

13. . Ibid., fol. $77 \mathrm{v}^{\circ}$.

14. . BnF ms fr. 22335, p. 143.

15. . Ibid., p. 249.

16. . Arch. nat., KK 85, fol. 97.

17. . Voir notamment Stéphane Castelluccio, Le Garde-Meuble de la Couronne et ses intendants du XVI ${ }^{\mathrm{e}}$ au XVIII ${ }^{\mathrm{e}}$ siècle, Bonchamp, éd. du CTHS, 2004. Pour l'auteur, «à la fin du XIV ${ }^{\mathrm{e}}$ siècle, la responsabilité des meubles royaux incombait au valet de chambre ». L'historien s'appuie sur le cas précis de Jean Moynat dit en 1387 « varlet de chambre et garde des chambres et tappis du roy [Charles VI] ». Le rôle du valet de chambre ainsi défini durerait jusqu'au XVIème siècle.

18. . Arch. nat., KK 82, fol. 49-50 v'.

19. . Arch. nat., KK 85, $97 \mathrm{r}^{\circ}$.

20. . Le Garde-Meuble royal, op. cit.

21. . BnF ms fr. 22335, p. 227.

22. . Notons que les concierges ne comptent pas parmi les officiers de l'hôtel de la reine.

23. . Les concierges perdurent jusqu'à la Révolution. Pour plus de précision sur l'histoire des concierges, voir Stéphane Castelluccio, Le Garde-Meuble de la Couronne, op. cit.

24. . BnF ms fr. 22335, p. 162. Le logis neuf dont il est question ici peut être soit le logis des Sept Vertus soit le logis royal face à la Loire, tous deux érigés à partir de 1494 et dans lesquels Anne de Bretagne a résidé. Voir à ce sujet Évelyne Thomas, «Les logis royaux d'Amboise », dans Revue de l'Art, 100, 1993.

25. . BnF ms fr. 22335, p. 187-190.

26. . Apparamment à l'occasion du décès de l'officier chargé de la garde-robe du roi mentionné comme « feu Pierre de garde robe », BnF ms fr. 22335, p. 190-191.

27. . BnF ms fr. 22335, p. 162 et 170 .

28. . Ibid., p. 125-127.

29. . Il semblerait que ce soit là une possession parisienne d'Anne de Bretagne et non l'hôtel parisien des rois Charles V et Charles VI. Dans les comptes de 1497-1498 il est en effet mentionné que la reine effectue des travaux dans « son hostel de Paris appelé la maison d'Estampes ».

30. . Le palais archiépiscopal de Lyon était la résidence principale des primats des Gaules au Moyen Âge. D'abord maison fortifiée, il est reconstruit à la fin du XV $\mathrm{XV}^{\mathrm{e}}$ siècle entre 1466 et 1467 par le cardinal Charles II de Bourbon, archevêque de Lyon, qui en fait un véritable palais.

31. . BnF ms fr. 22335, p. 127.

32. . Signalons notamment une salière de cristal, une autre de jaspe, un pot de cristal et plusieurs autres objets en argent doré.

33. . BnF ms fr. 22335, p. 209.

34. . La salle est la grande pièce qui compose le logis royal, qui jouxte le plus souvent la chambre. La garde-robe est une petite pièce qui jouxte elle aussi la chambre.

35. . Pour l'histoire de l'institution voir Stéphane Castelluccio, Le Garde-Meuble de la Couronne, op. cit.

36. . L'argentier, officier connu dans les sources à partir du XIV ${ }^{\mathrm{e}}$ siècle, avait pour rôle de fournir le roi et sa famille en mobilier et habillement.

37. . Louis Douët d'Arcq, " Notice sur les comptes de l'Argenterie », dans Comptes de l'argenterie des rois de France au XIV ${ }^{e}$ siècle, Paris, J. Renouard, 1866, p. VIII.

38. . Il est dit "garderobbier et argentier de ladite dame" dans les comptes de l'hôtel de 1492-1493. 
39. . Il est mentionné dans les inventaires de la reine à deux reprises seulement. Un inventaire est visiblement rédigé chez lui (BnF ms fr. 22335, p.112) et la nef de sainte Ursule lui a été temporairement confiée, à une date inconnue (BnF ms fr. 22335, p. 58).

40. . Probablement sa tâche se limite-t-elle à l'approvisionnement de la cour en meubles et habillement.

41. . Pour plus de précision sur le recours au château de Nantes comme garde-meuble personnel d'Anne de Bretagne, nous renvoyons à notre article «La gestion des collections d'Anne de Bretagne: une attraction bretonne", publié dans les actes à paraître du colloque "Nantes flamboyante (1380-1530) » qui s'est tenu à Nantes du 24 au 26 novembre 2011.

42. . Sont concernés des « relliques, relliquaires, ornemens d'esglise, paremens d'autel, tableaux, vaisselle d'or et d'argent, tappiceries de drap d'or, soye et layne, tappiz, acoustremens de escuierie, litz de camp, livres en latin, françoys, italien, grec et esbrieu, autres meubles et extencilles » (BnF ms fr. 22335, p. 53).

43. . BnF ms fr. 22335, p. 105.

44. . Cet inventaire est simplement mentionné mais il ne nous est pas parvenu (BnF ms fr. 22335, p. 112).

\section{RÉSUMÉS}

A la fin du XV ème siècle, la cour de France était itinérante et plusieurs des nombreuses résidences royales étaient concentrées en Val de Loire. Les déplacements d'un château à l'autre étaient fréquents et, à chaque fois, la cour emportait avec elle une bonne partie de ses effets, notamment ce qui relevait de l'ameublement intérieur, en vue de l'installation dans la résidence suivante. Il s'agit ici de voir comment s'organisait, en coulisses, cette itinérance des collections royales. Les inventaires d'Anne de Bretagne, successivement épouse des rois Charles VIII et Louis XII, fournissent, à ce sujet, de précieux renseignements qui rendent cette étude possible.

During the late Middle Ages, the royal court of France was itinerant. The numerous royal residencies were mainly concentrated along the Loire Valley, and there were very frequent moving from one castle to another. For each departure, the royal couple take with them part of their personal belongings, primarily pieces of furniture, in order to prepare their next stop. This work is concerned with the description of the behind the scenes organization of the royal collections travels and the profiling of the officials in charge of it. We believe that the inventories of queen Anne of Brittany, wife of kings Charles VIII (1483-1498) and Louis XII (1498-1515), provide some valuable information about the subject and are the foundations of our study.

Am Ende des Mittelalters fordert das Reisekönigtum, dass das königliche französische Ehepaar mit seiner Familie und seinem Hofstaat ständig von einer Pfalz zur anderen reist, welche zu dieser Epoche meistens am Loiretal lagen. Bei jeder Abreise nimmt der Hofstaat einen Teil seines Mobilars mit, um sich damit in dem nächsten Schloss einzurichten. Hier geht es darum, wie die Ortsveränderungen der königlichen Sammlungen hinter den Kulissen geleitet wurden. Was das Ende des 15. Jahrhunderts betrifft, liefern die in der Bibliothèque nationale de France aufbewahrten Bestandsverzeichnisse von Anne de Bretagne wertvolle Hinweise, die diese Forschung erst möglich machen. 


\section{AUTEUR}

\section{CAROLINE VRAND}

Née en 1986, Caroline Vrand est archiviste paléographe. Elle a soutenu sa thèse d'École nationale des chartes en 2010 : Les collections d'objets d'art d'Anne de Bretagne. Le spectacle et les coulisses, sous la direction de Philippe Plagnieux. Elle poursuit ses recherches sur les commandes artistiques d'Anne de Bretagne à l'université de Besançon dans le cadre d'une thèse de doctorat. Elle a publié plusieurs articles sur ce thème. Elle est actuellement conservateur-stagiaire à l'Institut national du patrimoine (spécialité musées).

carolinevrand@gmail.com 